\title{
SOME SHARP WEAK-TYPE INEQUALITIES FOR HOLOMORPHIC FUNCTIONS ON THE UNIT BALL OF $\mathrm{C}^{n}$
}

\author{
BOGUSLAW TOMASZEWSKI
}

Abstract. Let $B^{n}=\left\{z \in \mathbf{C}^{n}:|z|<1\right\}, S^{n}=\partial B^{n}$ and let $\sigma_{n}$ be the Haar measure on $S^{n}$. Then for all $f \in H^{p}(1 \leqslant p<\infty)$ such that $\operatorname{Im}(f(0))=0$ and $t>0$,

$$
\sigma_{n}\left(\left\{z \in S^{n}:|f(z)| \geqslant t\right\}\right) \leqslant C_{p} \cdot \frac{\|\operatorname{Re} f\|_{p}^{p}}{t^{p}}
$$

for some constant $C_{p}$ depending only on $p$. The best constant $C_{p}$ is found for $1 \leqslant p \leqslant 2$.

Let $\mathbf{C}^{n}$ be an $n$-dimensional complex space with norm

$$
\|z\|=\left(\left|z_{1}\right|^{2}+\cdots+\left|z_{n}\right|^{2}\right)^{1 / 2}
$$

and unit ball $B^{n}=\left\{z \in \mathbf{C}^{n}:\|z\|<1\right\}$. By $\sigma_{n}$ we shall denote the rotation-invariant, normalized Borel measure on $S^{n}=\partial B^{n}$. We shall write $D$ and $T$ instead of $B^{1}$ and $S^{1}$. For a $\sigma_{n}$-measurable function $f: S^{n} \rightarrow \mathrm{C}$ and $p \geqslant 1$, let us define

$$
\|f\|_{p}=\left(\int_{S^{n}}|f|^{p} d \sigma_{n}\right)^{1 / p} .
$$

If $\|f\|_{p}<\infty$ and if the Poisson integral $\mathrm{P}[f]$ of the function $f$ (see [5, p. 41]) is a holomorphic function, then we shall write $f \in H^{p}\left(S^{n}\right)$. Kolmogorov proved [4] that there exists a constant $C>0$ such that, if $f \in H^{1}(T)$ and if $f(0)=\mathrm{P}[f](0)$ is real, then

$$
\sigma_{1}(\{z \in T:|\operatorname{Im} f(z)| \geqslant t\}) \leqslant C \cdot \frac{\|\operatorname{Re} f\|_{1}}{t},
$$

for all $t>0$. In other words, the operator $\operatorname{Re} f \rightarrow \operatorname{Im} f$ is of the weak type 1-1 (see [7]). The best constant of inequality (1) was found by Davis [3]. Baernstein [2] gave an elementary proof of inequality (1) with the best constant. His proof was modified by Tomaszewski [6], who found the best constant in a weak-type inequality for the operator $\operatorname{Re} f \rightarrow f$. In this paper we shall prove similar sharp weak-type inequalities for spaces $H^{p}\left(S^{n}\right)$.

Received by the editors October 23, 1984 and, in revised form, December 28, 1984. The results of this paper were presented during the AMS meeting in San Diego, California, November 9-10, 1984. Presentation was sponsored by the Oklahoma State University.

1980 Mathematics Subject Classification. Primary 32-XX, 30-XX.

(1985 American Mathematical Society $0002-9939 / 85 \$ 1.00+\$ .25$ per page 
THEOREM. If $1 \leqslant p \leqslant 2, f \in H^{p}\left(S^{n}\right)$ and $\operatorname{Im} f(0)=0$, then for all $t>0$,

$$
\sigma_{n}\left(\left\{z \in S^{n}:|f(z)| \geqslant t\right\}\right) \leqslant C_{p} \cdot \frac{\|\operatorname{Re} f\|_{p}^{p}}{t^{p}},
$$

where

$$
C_{p}=\frac{\sqrt{\pi}}{2} \cdot \frac{p \cdot \Gamma(p / 2)}{\Gamma((p+1) / 2)} .
$$

The constant $C_{p}$ is the best possible in this inequality.

We shall need the following

Lemma. Let $u_{p}$, for $1 \leqslant p \leqslant 2$, be the Poisson integral of the function $\gamma_{p}\left(e^{i t}\right)=$ $|\cos t|^{p}$ defined on $T$. The inequalities

(i) $u_{p}(z) \leqslant u_{p}(0)+|\operatorname{Re} z|^{p}$,

(ii) $u_{p}(0) \leqslant u_{p}(x)$

hold for $z \in D$ and $-1 \leqslant x \leqslant 1$.

Proof. Let $u_{p}(z)=\operatorname{Re}\left(\sum_{k=0}^{\infty} a_{k} \cdot z^{k}\right)$ for some real numbers $a_{k}$. It is easy to see that $a_{2 n+1}=0$ for $n=0,1,2, \ldots$. We shall prove that

$$
(-1)^{n} \cdot a_{2 n} \leqslant 0
$$

for $n=1,2, \ldots$ We have

$$
\begin{aligned}
(-1)^{n} \cdot a_{2 n} & =\frac{(-1)^{n}}{\pi} \cdot \int_{-\pi}^{\pi}|\cos t|^{p} \cdot \cos 2 n t d t \\
& =\frac{1}{\pi} \cdot \int_{-\pi}^{\pi}|\sin t|^{p} \cdot \cos 2 n t d t \\
& =\frac{1}{\pi} \cdot \int_{-\pi}^{\pi}\left(1-\cos ^{2} t\right)^{p / 2} \cdot \cos 2 n t d t \\
& =\frac{1}{\pi} \cdot \sum_{m=1}^{\infty} b_{m} \cdot \int_{-\pi}^{\pi} \cos ^{2 m} t \cdot \cos 2 n t d t=\frac{1}{\pi} \cdot \sum_{m=1}^{\infty} b_{m} \cdot I_{2 m, 2 n},
\end{aligned}
$$

where $b_{m}<0$ are real numbers such that $(1-s)^{p / 2}=1+\sum_{m=1}^{\infty} b_{m} \cdot s^{m}$ and $I_{m, n}=$ $\int_{-\pi}^{\pi} \cos ^{n} t \cdot \cos m t d t$. Since $I_{m, n}=\frac{1}{2} \cdot\left(I_{m-1, n-1}+I_{m-1, n+1}\right)$ it can be easily proved (by induction on $m$ ) that $I_{m, n} \geqslant 0$. This ends the proof of inequality (3). Now, let us note that for $z=x+i y \in D$,

$$
\begin{aligned}
& u_{p}(z)-u_{p}(0)-a_{2} \cdot\left(x^{2}-y^{2}\right) \\
& \quad=\operatorname{Re}\left(\sum_{m=2}^{\infty} a_{2 m} \cdot z^{2 m}\right) \leqslant\left(x^{2}+y^{2}\right) \cdot \sum_{m=2}^{\infty}\left|a_{2 m}\right| \\
& \quad=\left(x^{2}+y^{2}\right) \cdot\left(u_{p}(0)-u_{p}(i)-a_{2}\right)=\left(x^{2}+y^{2}\right) \cdot \frac{2-p}{2 p} \cdot a_{2},
\end{aligned}
$$


since

$$
\begin{gathered}
a_{0}=\frac{2}{\pi} \cdot \frac{\Gamma((p+1) / 2) \cdot \Gamma(1 / 2)}{p \cdot \Gamma(p / 2)}, \\
a_{2}=\frac{1}{\pi} \cdot \int_{-\pi}^{\pi}|\cos t|^{p+2} d t-2 a_{0}=\frac{2 p}{p+2} \cdot a_{0} .
\end{gathered}
$$

But $(2-p) / 2 p \leqslant 1$ and

$$
x^{2} \cdot((2-p) / 2 p) \cdot a_{2}+x^{2} \cdot a_{2}=a_{0} \cdot x^{2} \leqslant x^{2} \leqslant x^{p} .
$$

Hence, inequality (i) follows. Let us turn to (ii). For $0 \leqslant \alpha \leqslant \pi / 2$ let $v_{\alpha}$ be the Poisson integral of the characteristic function, defined on $T$, of the set $\{z \in T$ : $|\operatorname{Im} z| \geqslant \sin \alpha\}$. Then

$$
v_{\alpha}(z)=\frac{1}{\pi} \cdot \operatorname{Arg}\left(\frac{1-i a-z-i a}{1-i a+z+i a}\right)+\frac{1}{\pi} \cdot \operatorname{Arg}\left(\frac{1+i a z+z-i a}{1+i a z-z+i a}\right)+1,
$$

where $a=(\cos \alpha) /(1+\sin \alpha)$. We see that

$$
v_{\alpha}(x)=\frac{2}{\pi} \cdot \operatorname{Arg}\left[1-a^{2}-i a\left(\frac{1+x}{1-x}+\frac{1-x}{1+x}\right)\right]+1 \leqslant v_{\alpha}(0) .
$$

But $u_{p}(z)=1-\int_{0}^{\pi / 2} v_{\alpha}(z) d \mu(\alpha)$ for some positive measure $\mu\left(d \mu(t)=p \cdot \cos ^{p-1} t\right.$ - $\sin t d t$ ). Thus, inequality (ii) follows.

Proof of The TheOrem. We shall first prove that equality holds in (2) for inner functions $f: S^{n} \rightarrow \mathrm{C}$ such that $f(0)=0$ (i.e. functions $f \in H^{p}\left(S^{n}\right)$ such that $|f(z)|$ $=1$ a.e. on $S^{n}$ and $\left.\int_{S^{n}} f(z) d \sigma_{n}(z)=0\right)$. The existence of such functions was proved by Aleksandrov [1]. If $f$ is inner and $f(0)=0$, then

$$
\int_{S^{n}} h(f(z)) d \sigma_{n}(z)=\int_{T} h(z) d \sigma_{1}(z),
$$

for every continuous function $h$ defined on $T$ (see $\left[5\right.$, p. 405]). Taking $h(z)=|\operatorname{Re} z|^{p}$, we see (2) cannot hold with any constant smaller than the constant $C_{p}$ defined above.

We shall prove inequality (2) for the case $n=1$. For each function $f \in H^{p}\left(S^{n}\right)$, we have

$$
\|\operatorname{Re} f\|_{p}^{p}=\int_{S^{n}} \int_{T}|\operatorname{Re} f(\xi z)|^{p} d \sigma_{1}(\xi) d \sigma_{n}(z) .
$$

If we now apply the statement of the Theorem, for the case $n=1$, to the integrals $\int_{T}|\operatorname{Re} f(\xi z)|^{p} d \sigma_{1}(\xi)$, we shall get the general case. Thus, let us assume that $f \in H^{p}(T)$. Let $\varphi: C \rightarrow R$ be a function such that $\varphi(z)=|\operatorname{Re} z|^{p}$ for $z \notin D$ and $\varphi(z)=u_{p}(z)$ for $z \in D$, where $u_{p}$ is defined in the lemma. The function $\varphi$ is continuous and since the function $|\operatorname{Re} z|^{p}$ is subharmonic, we have $\varphi(z) \geqslant|\operatorname{Re} z|^{p}$ for $z \in \mathbf{C}$. It follows that $\varphi$ is subharmonic on C. Let $E=\{z \in T:|f(z)| \geqslant 1\}$ and let us define the functions $\omega(z)=\mathrm{P}\left[\chi_{T \backslash E}\right](z), h(z)=\mathrm{P}\left[|\operatorname{Re} f|^{p}\right](z)$, where $\chi_{T \backslash E}$ is a characteristic function of the set $E$ and $\mathrm{P}$ denotes the Poisson integral for the unit disk $D$. We shall prove that

$$
\varphi(f(z)) \leqslant h(z)+\varphi(0) \cdot \omega(z)
$$


where we write $f(z)=\mathrm{P}[f](z)$. It suffices to check this inequality for $z \in T$, since the function $\varphi \circ f$ is subharmonic and the function $h(z)+\varphi(0) \cdot \omega(z)$ is harmonic. If $z \in T-E$, then $f(z) \in D$. Hence, for this case (4) follows from (i) and the definition of the function $h$ and the function $\omega$. If $z \in E$, then $\varphi(f(z))=|\operatorname{Re} f(z)|^{p}$ $=h(z)$, and (4) also holds for this case, hence for every $z \in T$. Taking $z=0$ in (4) and applying (ii), we get

$$
\begin{aligned}
\varphi(0) & \leqslant \varphi(f(0)) \leqslant h(0)+\varphi(0) \cdot \omega(0) \\
& =\|\operatorname{Re} F\|_{p}+\varphi(0) \cdot \sigma_{1}(T-E) .
\end{aligned}
$$

This ends the proof of inequality (2) for the case $t=1$, since $\sigma_{1}(T-E)=1-\sigma_{1}(E)$ and $\varphi(0)=u_{p}(0)=\left(C_{p}\right)^{-1}$. A general case can be proved by considering the function $f / t$ instead of $f$.

\section{REFERENCES}

1. A. B. Aleksandrov, The existence of inner functions in the ball, Mat. Sb. (N.S.) 118 (1982), 147-163 (Russian); Math. USSR-Sb. 43 (1983), 143-159.

2. A. Baernstein, Some sharp inequalities for conjugate functions, Indiana Univ. Math. J. 27 (1978), $833-852$.

3. B. Davis, On the weak-type (1-1) inequality for conjugate functions, Proc. Amer. Math. Soc. 44 (1974), 307-311.

4. A. N. Kolmogorov, Sur les fonctions harmoniques conjugées et la séries de Fourier, Fund. Math. 7 (1925), 23-28.

5. W. Rudin, Function theory in the unit ball of $\mathbf{C}^{n}$, Springer-Verlag, New York, 1980.

6. B. Tomaszewski, The best constant in a weak-type $H^{1}$-inequality, complex analysis and its applications, Complex Variables, Vol. 4, 1984, pp. 35-38.

7. A. Zygmund, Trigonometric series, Cambridge Univ. Press, London and New York, 1968.

Department of Mathematics, Oklahoma State University, Stillwater, Oklahoma 74078

Current address: Department of Mathematics, State University of New York, 1400 Washington Avenue, Albany, New York 12222 